\title{
A FORMULA RELATING INFLECTIONS, BITANGENCIES AND THE MILNOR NUMBER OF A PLANE CURVE
}

\author{
FABIO SCALCO DIAS, RAÚL OSET SINHA, AND MARIA APARECIDA SOARES RUAS
}

(Communicated by Lev Borisov)

\begin{abstract}
In this article we obtain a formula relating inflections, bitangencies and the Milnor number of a plane curve germ. Moreover, we present an extension of the formula obtained by the first author and Luis Fernando Mello for a class of plane curves with singularities.
\end{abstract}

\section{INTRODUCTION}

The study of plane curves goes far back in history. This study has been carried out from two different points of view, the geometrical one and the algebraic one. In this article we prove different formulae from the geometric and algebraic points of view, but using a common tool. More precisely, given $\gamma: I \rightarrow \mathbb{R}^{2}$ where $I$ is an open interval $\left(\gamma:(\mathbb{C}, 0) \rightarrow\left(\mathbb{C}^{2}, 0\right)\right.$ for the complex case $)$, we define the vector field $F_{\gamma}: \mathbb{R} \times \mathbb{R} \rightarrow \mathbb{R}^{2}\left(F_{\gamma}:(\mathbb{C} \times \mathbb{C}, 0) \rightarrow\left(\mathbb{C}^{2}, 0\right)\right)$ as

$$
F_{\gamma}(u, v)=\left(\frac{\operatorname{det}\left(\gamma^{\prime}(u), \gamma(u)-\gamma(v)\right)}{(u-v)^{2}}, \frac{\operatorname{det}\left(\gamma^{\prime}(v), \gamma(u)-\gamma(v)\right)}{(u-v)^{2}}\right) \text {. }
$$

Using this vector field we study relations between different invariants both in the geometric (real) case and in the algebraic (complex) case.

The paper is organized as follows. Section 2 gives the necessary preliminaries for Sections 3 and 4 .

For Section 3, consider the following setting: Let $\gamma: I \rightarrow \mathbb{R}^{2}, \gamma(u)=\left(\gamma_{1}(u), \gamma_{2}(u)\right)$ be a smooth plane curve, with $I \subset \mathbb{R}$ an open interval. A bitangency is a pair of points $u, v \in I$, denoted by $(u, v)$, such that the tangent line of $\gamma$ at $u$ coincides with the tangent line of $\gamma$ at $v$. A bitangency $(u, v)$ is a same side bitangency if $\gamma$ near $u$ and $\gamma$ near $v$ lie on the same side of the common tangent line (see Figure1(a)), and a bitangency is an opposite side bitangency if $\gamma$ near $u$ and $\gamma$ near $v$ lie on opposite sides of the common tangent line (see Figure 1 (b)). Analogously a tritangency is defined by the points $u, v, w \in I$ such that the tangent lines of $\gamma$ at $u, v$ and $w$ coincide. A double point is a pair of points $u, v \in I$, denoted by $(u, v)$, such that $\gamma(u)=\gamma(v)$, that is, a self-intersection of the curve (see Figure 1 (c)), and a triple point is defined by the points $u, v, w \in I$ such that $\gamma(u)=\gamma(v)=\gamma(w)$.

Received by the editors November 11, 2011 and, in revised form, August 5, 2012.

2010 Mathematics Subject Classification. Primary 14H20; Secondary 53A55, 58K60.

Key words and phrases. Milnor number, plane curves, double point, inflection, bitangency.

The first author was supported by FAPESP grant No. 2011/01946-0.

The second author was partially supported by FAPESP grant No. 2010/01501-5 and DGCYT and FEDER grant No. MTM2009-08933.

The third author was supported by FAPESP, grant No. 08/54222-6 and CNPq, grant No. 303774/2008-8. 
An inflection point is a point $u \in I$ such that the curvature of $\gamma$ at $u$ vanishes, i.e. $k_{\gamma}(u):=\frac{\operatorname{det}\left(\gamma^{\prime}(u), \gamma^{\prime \prime}(u)\right)}{\left\|\gamma^{\prime}(u)\right\|}=0$ (see Figure 1 $(\mathrm{d})$ ).

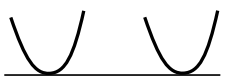

(a)

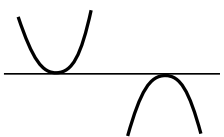

(b)

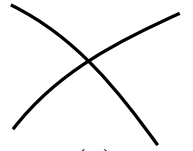

(c)

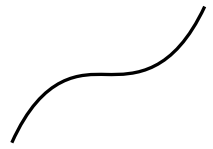

(d)

Figure 1. (a) same side bitangency, (b) opposite side bitangency,

(c) double point and (d) inflection point.

A cusp such that the two parts of the curve adjacent to the cusp lie on opposite sides (on the same side) of the limiting tangent to the curve at the cusp is said to be a cusp of the first kind (second kind).

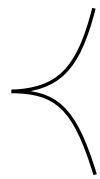

(a)

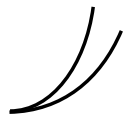

(b)

Figure 2. (a) cusp of the first kind, (b) cusp of the second kind.

For a given plane curve, denote by $t_{s}$ and $t_{o}$ the numbers of the same side and opposite side bitangencies respectively, by $d$ the number of double points, by $i$ the number of inflections and by $c_{1}$ and $c_{2}$ the number of cusps of the first and second kinds, respectively.

Given a closed curve $\gamma: \mathbb{S}^{1} \rightarrow \mathbb{R}^{2}$, Fabricius-Bjerre [7] proved, considering a regularity condition slightly stronger than $C^{1}$, that

$$
t_{s}-t_{o}=d+\frac{i}{2}
$$

Later, Fabricius-Bjerre 8 gave a proof of an equation similar to (1.1) for curves with cusps. Halpern [10] proved (1.1) for smooth closed curves satisfying other regularity conditions. His proof applies to curves of class $C^{4}$ and relies on techniques based on winding numbers and singular points of vector fields. Weiner [17] generalized the formula to closed curves lying on a 2-sphere. Ferrand [9] related the Fabricius-Bjerre and Weiner formulas to Arnold's invariants for plane curves. Dias and Mello [4] extended equation (1.1) for a class of plane curves $\gamma: \mathbb{R} \rightarrow \mathbb{R}^{2}$.

In this paper, we obtain formulae involving these numbers for deformations of a certain class of plane curves $\mathcal{G}^{\prime}\left(I, \mathbb{R}^{2}\right.$ ) (see Section 3 ). More specifically, we prove the following theorem, which is an extension of a theorem in [4].

Theorem 3.10. Let $\alpha(u)=\left(a_{k} u^{k}, b_{m} u^{m}\right)$ be a plane curve where $0<k<m$, $a_{k} b_{m} \neq 0$. For any generic deformation of $\alpha$ the following statements hold:

i) If $k$ and $m$ are either both even or both odd, then

$$
t_{s}-t_{o}=d+c_{1}+\frac{i-1+c_{2}}{2} .
$$


ii) If $k$ is even and $m$ is odd, then

$$
t_{s}-t_{o}=d+c_{1}+\frac{i+c_{2}}{2}-1
$$

iii) If $k$ is odd and $m$ is even, then

$$
t_{s}-t_{o}=d+c_{1}+\frac{i+c_{2}}{2} .
$$

In Section 4 , we consider the germ of an irreducible plane curve $\gamma:(\mathbb{C}, 0) \rightarrow$ $\left(\mathbb{C}^{2}, 0\right)$. We then define

\section{Definition 1.1.}

i)

$$
I:=\operatorname{dim}_{\mathbb{C}} \frac{\mathcal{O}_{\mathbb{C}, 0}}{\left\langle\operatorname{det}\left(\gamma^{\prime}(u), \gamma^{\prime \prime}(u)\right)\right\rangle} \quad \text { and } \quad \tilde{I}:=\operatorname{dim}_{\mathbb{C}} \frac{\mathbb{C}[u]}{\left\langle\operatorname{det}\left(\gamma^{\prime}(u), \gamma^{\prime \prime}(u)\right)\right\rangle} ;
$$

ii)

$$
\delta:=\frac{1}{2} \operatorname{dim}_{\mathbb{C}} \frac{\mathcal{O}_{\mathbb{C}^{2}, 0}}{\left\langle\frac{\gamma_{1}(u)-\gamma_{1}(v)}{u-v}, \frac{\gamma_{2}(u)-\gamma_{2}(v)}{u-v}\right\rangle} \quad \text { and } \quad \tilde{\delta}:=\frac{1}{2} \operatorname{dim}_{\mathbb{C}} \frac{\mathbb{C}[u, v]}{\left\langle\frac{\gamma_{1}(u)-\gamma_{1}(v)}{u-v}, \frac{\gamma_{2}(u)-\gamma_{2}(v)}{u-v}\right\rangle} .
$$

The numbers $I$ and $\delta$ are the number of inflection points and of double points of a deformation of the curve respectively, and $\tilde{I}$ and $\tilde{\delta}$ are the global number of inflections and double points (see Section 4 ). Let $T$ be the number of bitangencies of a deformation of the curve and let $\tilde{T}$ be $T$ plus the number of bitangencies of the curve before the deformation. We give a relation between the number of inflections, double points and bitangencies and then we prove the following:

Theorem 4.4. Consider $\gamma$ a plane curve germ

$$
\gamma(u)=\left(\sum_{i=k}^{l} a_{i} u^{i}, \sum_{j=m}^{n} b_{j} u^{j}\right)
$$

with $a_{k} a_{l} b_{m} b_{n} \neq 0$ and $1 \leq k<m$. Then

i) If $k$ and $m$ are co-prime, then

$$
\mu=I(I-1)-2 T
$$

ii) If $l$ and $n$ are co-prime, then

$$
\tilde{\mu}=\tilde{I}(\tilde{I}-1)-2 \tilde{T},
$$

where $\mu$ and $\tilde{\mu}$ are the local and global Milnor numbers of the plane curve germ.

Results similar to Theorem 4.4 have not been found in the literature. A good account on plane curves can be found in [16]. The techniques used in the article to obtain Theorem 4.4 strongly use the fact that the degrees are $k, l, m, n$. The general case is probably too broad for this type of result. However, it seems likely that there may be similar formulae for certain specific classes of plane curves. 


\section{Preliminaries}

2.1. Vector fields. Let $X: U \rightarrow \mathbb{R}^{n}$ be a vector field, where $U$ is an open set of $\mathbb{R}^{n}$. Let $q$ be an isolated critical point of $X$ in $U$. Then the function $X(u) /\|X(u)\|$ maps a small sphere, centered at $q$, into the unit $(n-1)$-sphere. It is well known that the degree of this mapping is called the index of $X$ at $q$ and is denoted by $\operatorname{ind}_{q}(X)$ or $i(q)$. In the case of a vector field $X$ on a manifold $M$, we define the index of $X$ at an isolated zero $x_{0}$ through a local parametrization.

The topological index of $X, \operatorname{ind}(X)$, is an integer such that if $X$ has finitely many critical points $p_{1}, p_{2}, \ldots, p_{k}$, then

$$
i n d(X)=\sum_{j=1}^{k} i n d_{p_{j}}(X) .
$$

In this context we can state the following well known result; see [11] or [12].

Proposition 2.1. Let $M, N$ be oriented $n$-manifolds without boundary, with $M$ compact and $N$ connected. Assume that $X, Y: M \rightarrow N$ are homotopic; that is, there is a continuous map $H: M \times[0,1] \rightarrow N$ with $H(x, 0)=X(x)$ and $H(x, 1)=Y(x)$. Then $\operatorname{ind}(X)=\operatorname{ind}(Y)$.

In order to study the behaviour of the trajectories of a polynomial planar vector field near infinity, it is possible to use a compactification. One of the possible constructions relies on the stereographic projection of the sphere onto the plane, in which case a single point at infinity is adjoined to the plane. More specifically, let $\mathbb{S}^{2}$ be the two-sphere in $\mathbb{R}^{3}$ defined by the set $\left\{y \in \mathbb{R}^{3}: y_{1}^{2}+y_{2}^{2}+\left(y_{3}-\frac{1}{2}\right)^{2}=1 / 4\right\}$, known as the Bendixson sphere. The plane $\mathbb{R}^{2}$ may be identified with the sphere $\mathbb{S}^{2}$ with the "north pole" $p=(0,0,1)$ removed. We shall call the Bendixson compactification of $X, s(X)$, the induced vector field in the two-sphere; for more details, see [6]. Notice that the behaviour of the orbits of $X$ near infinity is determined by the behaviour of $s(X)$ near $p=(0,0,1)$. The point $p$ is called the infinite critical point of $s(X)$. We shall refer to the critical points of $s(X)$ which are in $\mathbb{S}^{2} \backslash p$ as finite critical points of $X$ or $s(X)$. It follows from equation (2.1) that

$$
\operatorname{ind}(s(X))=\operatorname{ind}(X)+\operatorname{ind}_{p}(s(X)) .
$$

2.2. Bézout's Theorem and other algebraic considerations. In Section 4 we will need the following result. Suppose we have $n$ homogeneous polynomials $F_{1}, \ldots, F_{n}$ of degree $d_{1}, \ldots, d_{n}$ in complex variables $x_{0}, \ldots, x_{n}$. Define

$$
\begin{aligned}
& f_{i}\left(x_{1}, \ldots, x_{n}\right)=F_{i}\left(1, x_{1}, \ldots, x_{n}\right), \\
& \bar{F}_{i}\left(x_{1}, \ldots, x_{n}\right)=F_{i}\left(0, x_{1}, \ldots, x_{n}\right) .
\end{aligned}
$$

Note that $f_{i}$ has total degree at most $d_{i}$. Inside $\mathbb{P}^{n}$, we have the affine space $\mathbb{C}^{n} \subset \mathbb{P}^{n}$ defined by $x_{0}=1$, and the solutions of the affine equations

$$
f_{1}=\cdots=f_{n}=0
$$

are precisely the solutions of $F_{1}=\cdots=F_{n}=0$ which lie in $\mathbb{C}^{n}$. Similarly, the nontrivial solutions of the homogeneous equations

$$
\bar{F}_{1}=\cdots=\bar{F}_{n}=0
$$

may be regarded as the solutions which lie "at $\infty$ ". We say that (2.4) has no solutions at $\infty$ if $\bar{F}_{1}=\cdots=\bar{F}_{n}=0$ has no nontrivial solutions. 
Theorem 2.2 (Bézout's Theorem). Assume that $f_{1}, \ldots, f_{n}$ are defined as in (2.3) and that the affine equations (2.4) have no solution at $\infty$. Then these equations have $d_{1} \cdots d_{n}$ solutions (counted with multiplicity), and

$$
\operatorname{dim}_{\mathbb{C}} \frac{\mathbb{C}\left[x_{1}, \ldots, x_{n}\right]}{\left\langle f_{1}, \ldots, f_{n}\right\rangle}=d_{1} \cdots d_{n}
$$

as a vector space over $\mathbb{C}$.

The proof of Theorem 2.2 may be found in 3 .

To define the local multiplicity of a solution of a system of equations, we use the local ring instead of the polynomial ring, but the idea is much the same. Consider $f:\left(\mathbb{C}^{n}, 0\right) \rightarrow\left(\mathbb{C}^{n}, 0\right)$ to be a holomorphic map germ at the origin. The multiplicity of the germ $f$ at the origin is given by

$$
\mu_{0}[f]=\operatorname{dim}_{\mathbb{C}} \frac{\mathcal{O}_{\mathbb{C}^{n}, 0}}{\langle f\rangle},
$$

where $\mathcal{O}_{\mathbb{C}^{n}, 0}$ is the ring of germs at 0 of smooth complex-valued functions on $\mathbb{C}^{n}$, and $\langle f\rangle$ is the ideal generated by the components of $f$. We say that $f$ is a finite map germ if $\mu_{0}[f]<\infty$. It is known that the multiplicity of $f$ is the number of preimages of a regular value of $f$ near 0 .

Remark 2.3.

$$
\operatorname{dim}_{\mathbb{C}} \frac{\mathbb{C}\left[x_{1}, \ldots, x_{n}\right]}{\left\langle f_{1}, \ldots, f_{n}\right\rangle}=\sum_{p \in f^{-1}(0)} \operatorname{dim}_{\mathbb{C}} \frac{\mathcal{O}_{\mathbb{C}^{n}, p}}{\left\langle f_{1}, \ldots, f_{n}\right\rangle},
$$

where $f=\left(f_{1}, \ldots, f_{n}\right)$ is finite. Therefore, the dimension of the quotient ring $\mathbb{C}\left[x_{1}, \ldots, x_{n}\right] /\left\langle f_{1}, \ldots, f_{n}\right\rangle$ is the number of points of $f_{1}=\cdots=f_{n}=0$ counted with multiplicity. On the other hand, the dimension of $\mathcal{O}_{\mathbb{C}^{n}, p} /\left\langle f_{1}, \ldots, f_{n}\right\rangle$ is the multiplicity of $p$.

Consider $f:\left(\mathbb{C}^{n}, 0\right) \rightarrow\left(\mathbb{C}^{n}, 0\right)$, where $f=\left(f_{1}, \ldots, f_{n}\right)$ with each $f_{i}$ a homogeneous polynomial such that 0 is isolated in $f^{-1}(0)$. It is well known that $\mu_{0}[f]=\Pi_{i=1}^{n} d_{i}$, where $d_{i}$ is the degree of each $f_{i}$. On the other hand any holomorphic function $f_{i}:\left(\mathbb{C}^{n}, 0\right) \rightarrow(\mathbb{C}, 0)$ can be written as $f_{i}=g_{i}+G_{i}$, where $g_{i}$ is the homogeneous polynomial of lowest degree possible in $f_{i}$. Hence any smooth map $f:\left(\mathbb{C}^{n}, 0\right) \rightarrow\left(\mathbb{C}^{n}, 0\right)$ can be written as $f=g+G$. It is also known that $\mu_{0}[f]=\mu_{0}[g]$ if 0 is isolated in $g^{-1}(0)$. See [1] for an example.

\section{A GeOMETRIC FORMUlA}

In [4] the authors defined $\mathcal{G}\left(I, \mathbb{R}^{2}\right)$ as the set of $C^{\infty}$ plane curves $\gamma: I \rightarrow \mathbb{R}^{2}$ satisfying the following conditions:

1) The curve $\gamma$ has a finite number of transversal double points; that is, if $(u, v)$ is a double point, then $\gamma^{\prime}(u)$ is not a multiple of $\gamma^{\prime}(v)$ and there are no triple points.

2) The curve $\gamma$ has a finite number of bitangencies and there are no tritangencies.

3) The curve $\gamma$ has a finite number of ordinary inflection points; that is, if $\kappa_{\gamma}(u)=0$, then $\kappa_{\gamma}^{\prime}(u) \neq 0$, and an inflection point is not part of a bitangency. 
Definition 3.1 ([4]). Let $\gamma(u)=\left(a_{k} u^{k}, b_{m} u^{m}\right)$ be a plane curve with $0<k<m$ and $a_{k} b_{m} \neq 0$. A deformation in $\mathcal{G}\left(I, \mathbb{R}^{2}\right)$ of $\gamma$ is a plane curve

$$
\bar{\gamma}(u)=\left(a_{k} u^{k}+a_{k-1} u^{k-1}+\ldots+a_{1} u, b_{m} u^{m}+b_{m-1} u^{m-1}+\ldots+b_{1} u\right)
$$

such that $\bar{\gamma} \in \mathcal{G}\left(I, \mathbb{R}^{2}\right)$.

Theorem 3.2 (4]). Let $\gamma(u)=\left(a_{k} u^{k}, b_{m} u^{m}\right)$ be a plane curve with $0<k<m$ and $a_{k} b_{m} \neq 0$. For any deformation in $\mathcal{G}\left(I, \mathbb{R}^{2}\right)$ of $\gamma$ the following statements hold:

i) If $k$ and $m$ are either both even or both odd, then

$$
t_{s}-t_{o}=d+\frac{i-1}{2} .
$$

ii) If $k$ is even and $m$ is odd, then

$$
t_{s}-t_{o}=d+\frac{i}{2}-1
$$

iii) If $k$ is odd and $m$ is even, then

$$
t_{s}-t_{o}=d+\frac{i}{2}
$$

Here we prove an extension of this theorem for a bigger set of plane curves and a different type of deformation. We consider the set $\mathcal{G}^{\prime}\left(I, \mathbb{R}^{2}\right)$ of plane curves which satisfy 1 ) and 2) of $\mathcal{G}\left(I, \mathbb{R}^{2}\right)$ plus the following conditions:

$3^{\prime}$ ) The curve $\gamma$ has a finite number of ordinary inflection points; that is, if $\kappa_{\gamma}(u)=0$, then $\kappa_{\gamma}^{\prime}(u) \neq 0$, where $u$ is not a cusp, and an inflection point is not part of a bitangency.

4) The curve $\gamma$ has a finite number of cusps of the first and second kind.

By using standard transversality techniques, it is possible to show that the set $\mathcal{G}^{\prime}\left(I, \mathbb{R}^{2}\right) \subset C^{\infty}\left(I, \mathbb{R}^{2}\right)$ is residual with the Whitney $C^{\infty}$ topology.

Let $\gamma: I \rightarrow \mathbb{R}^{2} \in \mathcal{G}^{\prime}\left(I, \mathbb{R}^{2}\right)$. Define the vector field $F_{\gamma}: I \times I \rightarrow \mathbb{R}^{2}$ as

$$
F_{\gamma}(u, v)=\left(\frac{\operatorname{det}\left(\gamma^{\prime}(u), \gamma(u)-\gamma(v)\right)}{(u-v)^{2}}, \frac{\operatorname{det}\left(\gamma^{\prime}(v), \gamma(u)-\gamma(v)\right)}{(u-v)^{2}}\right) .
$$

The following proposition establishes a relationship between the zeros of $F_{\gamma}$ and the geometry of the curve $\gamma$.

Proposition 3.3. Consider $\gamma \in \mathcal{G}^{\prime}\left(I, \mathbb{R}^{2}\right)$ and $F_{\gamma}$ as in (3.1). Then $F_{\gamma}$ is welldefined in $u=v$ and

$F_{\gamma}(u, v)=(0,0) \Leftrightarrow \begin{cases}(u, v) \text { is a double point or a bitangency of } \gamma, & \text { when } u \neq v ; \\ v \text { is a inflection of } \gamma, & \text { when } u=v ; \\ v \quad \text { is a cusp of } \gamma, & \text { when } u=v .\end{cases}$

Proof. The proof of this proposition, except for the last item, can be found in [4]. It is evident that $F_{\gamma}(u, v)=(0,0)$ if and only if $(u, v)$ is a bitangency or a double point when $u \neq v$. In what follows we prove $F_{\gamma}$ is well-defined when $u=v$. Consider $f(u, v)=\operatorname{det}\left(\gamma^{\prime}(u), \gamma(u)-\gamma(v)\right) /(u-v)^{2}$. Thus $F_{\gamma}(u, v)=(f(u, v),-f(v, u))$. Expanding $\gamma(u)$ and $\gamma^{\prime}(u)$ into a Taylor series with center at $u=v$, we have

$$
\frac{\gamma(u)-\gamma(v)}{u-v}=\gamma^{\prime}(v)+\frac{\gamma^{\prime \prime}(v)}{2 !}(u-v)+\frac{\gamma^{\prime \prime \prime}(v)}{3 !}(u-v)^{2}+\ldots
$$

and

$$
\gamma^{\prime}(u)=\gamma^{\prime}(v)+\gamma^{\prime \prime}(v)(u-v)+\frac{\gamma^{\prime \prime \prime}(v)}{2 !}(u-v)^{2}+\ldots
$$


Hence

$$
\begin{aligned}
& f(u, v) \\
& =\frac{\operatorname{det}\left(\gamma^{\prime}(v)+\gamma^{\prime \prime}(v)(u-v)+\frac{\gamma^{\prime \prime \prime}(v)}{2 !}(u-v)^{2}+\cdots, \gamma^{\prime}(v)+\frac{\gamma^{\prime \prime}(v)}{2 !}(u-v)+\frac{\gamma^{\prime \prime \prime}(v)}{3 !}(u-v)^{2}+\cdots\right)}{(u-v)} \\
& =\frac{3 \operatorname{det}\left(\gamma^{\prime}(v), \gamma^{\prime \prime}(v)\right)}{2}+(u-v) \tilde{f}(u, v) .
\end{aligned}
$$

Taking $u=v$ it follows that

$$
F_{\gamma}(u, u)=\left(\frac{3 \operatorname{det}\left(\gamma^{\prime}(u), \gamma^{\prime \prime}(u)\right)}{2},-\frac{3 \operatorname{det}\left(\gamma^{\prime}(u), \gamma^{\prime \prime}(u)\right)}{2}\right) .
$$

Therefore, $F_{\gamma}(u, u)$ is well-defined and $F_{\gamma}(u, u)=0$ if and only if $\operatorname{det}\left(\gamma^{\prime}(u), \gamma^{\prime \prime}(u)\right)=$ 0 ; that is, $u$ is an inflection point or a cusp $\left(\gamma^{\prime}(u)=0\right)$.

Proposition 3.4. Consider $\gamma \in \mathcal{G}^{\prime}\left(I, \mathbb{R}^{2}\right)$.

i) The index $\operatorname{ind}_{p}\left(F_{\gamma}\right)$ is given by

$$
\operatorname{ind}_{p}\left(F_{\gamma}\right)= \begin{cases}-1, & \text { at a double point of } \gamma ; \\ -1, & \text { at an opposite side bitangency of } \gamma ; \\ +1, & \text { at a same side bitangency of } \gamma ; \\ -1, & \text { at an inflection of } \gamma ; \\ -2, & \text { at a first kind cusp of } \gamma ; \\ -1, & \text { at a second kind cusp of } \gamma\end{cases}
$$

ii) The index $\operatorname{ind}\left(F_{\gamma}\right)$ is given by

$$
\operatorname{ind}\left(F_{\gamma}\right)=-2 d-2 t_{o}+2 t_{s}-i-2 c_{1}-c_{2} .
$$

Proof. i) See Theorem 2.3 in [4]. For the indices of cusps, see Theorem 3.5 in [4] (which is stated here as Theorem 3.8).

ii) It follows from the definition of $F_{\gamma}$ that $F_{\gamma}(u, v)=(0,0)$ if and only if $F_{\gamma}(v, u)=(0,0)$. Thus the proof follows from equation (2.1) and item i).

For the calculation of $\operatorname{ind}\left(F_{\gamma}\right)$ in equation (3.2) we need to consider a plane curve $\alpha: I \rightarrow \mathbb{R}^{2}$ of the form

$$
\alpha(u)=\left(a_{k} u^{k}, b_{m} u^{m}\right),
$$

where $0<k<m, a_{k} b_{m} \neq 0$ and $I \subset \mathbb{R}$ is an open interval containing the origin. We define the associated vector field $F_{\alpha}$ as in (3.1).

Remark 3.5. In the case $(k, m)=$ (Even, Even) we consider the vector field $F_{\alpha} /(u+$ $v)^{2}$ instead of $F_{\alpha}$. For simplicity this new vector field will be denoted by $F_{\alpha}$ too.

Definition 3.6. We say that a plane curve $\gamma \in \mathcal{G}^{\prime}\left(I, \mathbb{R}^{2}\right)$ is a generic deformation of $\alpha$ as in (3.3) if

i) There is a continuous homotopy $H: I \times[0,1] \rightarrow \mathbb{R}^{2}$ such that

$$
\begin{aligned}
& H(u, 0)=\gamma(u) \text { and } \\
& H(u, 1)=\alpha(u) .
\end{aligned}
$$

ii) There exists $\delta \gg 0$ such that for all $s$, the subset $\left\{F_{H_{s}(u)}=0\right\} \subset B_{\delta}$. 
Example 3.7. Consider the homotopy $H$ between the curves $\alpha(u)=\left(u, u^{3}\right)$ and $\gamma(u)=\left(u^{2}, u^{3}\right)$, given by $H(u, s)=\left((1-s) u+s u^{2}, u^{3}\right)$. The inflection points of the curve $H_{s}(u)$ are given by

$$
u=0 \text { and } u=\frac{s-1}{s} .
$$

Thus, there is no $\delta$ such that $\left\{F_{H_{s}(u)}=0\right\} \subset B_{\delta}$ when $s \rightarrow 0$.

Theorem 3.8. Let $\alpha(u)=\left(a_{k} u^{k}, b_{m} u^{m}\right)$ be a plane curve where $0<k<m$, $a_{k} b_{m} \neq 0$. Then

$$
\operatorname{ind}\left(F_{\alpha}\right)=\left\{\begin{aligned}
-1, & \text { if }(k, m)=(\text { Even }, \text { Even }) \\
-2, & \text { if }(k, m)=\text { (Even, Odd) } \\
0, & \text { if }(k, m)=\text { (Odd, Even) } \\
-1, & \text { if }(k, m)=(\text { Odd }, \text { Odd })
\end{aligned}\right.
$$

Proof. See 4 .

Lemma 3.9. Consider $\alpha$ as above and $\gamma$ a generic deformation of $\alpha$. Then

$$
\operatorname{ind}\left(F_{\alpha}\right)=\operatorname{ind}\left(F_{\gamma}\right) \text {. }
$$

Proof. It is easy to see that the homotopy between the curves $\alpha$ and $\gamma$ induces a homotopy between the vector fields $F_{\alpha}$ and $F_{\gamma}$. Consider $s\left(F_{\alpha}\right): \mathbb{S}^{2} \rightarrow \mathbb{R}^{2}$ and $s\left(F_{\gamma}\right): \mathbb{S}^{2} \rightarrow \mathbb{R}^{2}$, the Bendixson compactification of the vector fields $F_{\alpha}$ and $F_{\gamma}$, respectively. It follows from Proposition 2.1 that $\operatorname{ind}\left(s\left(F_{\alpha}\right)\right)=\operatorname{ind}\left(s\left(F_{\gamma}\right)\right)$. Equation (2.2) implies that

$$
\operatorname{ind}\left(F_{\alpha}\right)+\operatorname{ind}_{p}\left(s\left(F_{\alpha}\right)\right)=\operatorname{ind}\left(s\left(F_{\alpha}\right)\right)=\operatorname{ind}\left(s\left(F_{\gamma}\right)\right)=\operatorname{ind}\left(F_{\gamma}\right)+\operatorname{ind} d_{p}\left(s\left(F_{\gamma}\right)\right),
$$

where $\operatorname{ind}_{p}\left(s\left(F_{\alpha}\right)\right)$ denotes the index of $s\left(F_{\alpha}\right)$ at the infinite critical point $p$. Therefore,

$$
\operatorname{ind}\left(F_{\alpha}\right)+\operatorname{ind}_{p}\left(s\left(F_{\alpha}\right)\right)=\operatorname{ind}\left(F_{\gamma}\right)+i n d_{p}\left(s\left(F_{\gamma}\right)\right) .
$$

It can be seen that $F_{\alpha}$ has no critical point at infinity. On the other hand, it follows from condition ii) of Definition 3.6 that $F_{\gamma}$ does not have infinite critical points either. Thus, $\operatorname{ind}_{p}\left(s\left(F_{\alpha}\right)\right)=i n d_{p}\left(s\left(F_{\gamma}\right)\right)=0$, and therefore

$$
\operatorname{ind}\left(F_{\alpha}\right)=\operatorname{ind}\left(F_{\gamma}\right) \text {. }
$$

Theorem 3.10. Let $\alpha(u)=\left(a_{k} u^{k}, b_{m} u^{m}\right)$ be a plane curve where $0<k<m$, $a_{k} b_{m} \neq 0$. For any generic deformation of $\alpha$ the following statements hold:

i) If $k$ and $m$ are either both even or both odd, then

$$
t_{s}-t_{o}=d+c_{1}+\frac{i-1+c_{2}}{2} .
$$

ii) If $k$ is even and $m$ is odd, then

$$
t_{s}-t_{o}=d+c_{1}+\frac{i+c_{2}}{2}-1 .
$$

iii) If $k$ is odd and $m$ is even, then

$$
t_{s}-t_{o}=d+c_{1}+\frac{i+c_{2}}{2} .
$$


Proof. Consider $\alpha(u)=\left(a_{k} u^{k}, b_{m} u^{m}\right)$. By Theorem 3.8 the associated vector field $F_{\gamma}$ has index -1 if either $(k, m)=$ (Even, Even) or $(k, m)=$ (Odd, Odd), -2 if $(k, m)=($ Even, Odd $)$, and 0 if $(k, m)=($ Odd, Even $)$.

Let $\gamma$ be a generic deformation of $\alpha$. From Proposition 3.4 the corresponding vector field $F_{\gamma}$ has index given by $-2 d-2 t_{o}+2 t_{s}-i-2 c_{1}-c_{2}$ (see equation (3.2)). On the other hand, it follows from Lemma 3.9 that $\operatorname{ind}\left(F_{\alpha}\right)=\operatorname{ind}\left(F_{\gamma}\right)$. Hence, we have the following cases:

Case i) $(k, m)=($ Even, Even $)$ or $(k, m)=($ Odd, Odd $)$. Thus

$$
-2 d-2 t_{o}+2 t_{s}-i-2 c_{1}-c_{2}=\operatorname{ind}\left(F_{\alpha}\right)=-1,
$$

which is equivalent to equation (3.4).

Case ii) $(k, m)=($ Even, Odd $)$. In this case

$$
-2 d-2 t_{o}+2 t_{s}-i-2 c_{1}-c_{2}=i n d\left(F_{\alpha}\right)=-2,
$$

which is equivalent to equation (3.5).

Case iii) $(k, m)=($ Odd, Even $)$. In this case

$$
-2 d-2 t_{o}+2 t_{s}-i-2 c_{1}-c_{2}=i n d\left(F_{\alpha}\right)=0,
$$

which is equivalent to equation (3.6).

An example of a plane curve with $k=2$ and $m=5$ is illustrated in Figure 3 ,

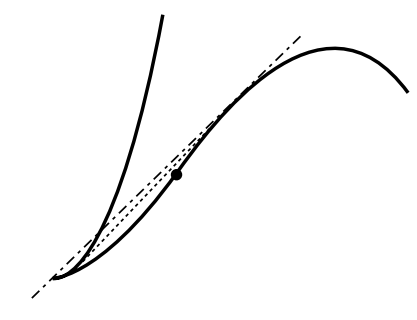

Figure 3. $\gamma(u)=\left(u^{2}, u^{4}+u^{5}\right), t_{o}=t_{s}=i=c_{2}=1$, and $d=c_{1}=0$.

\section{An Algebraic formula}

In this section we use the vector field $F_{\gamma}$ to obtain a formula relating inflections, bitangencies and the Milnor number of plane curve germs.

Let $\gamma:(\mathbb{C}, 0) \rightarrow\left(\mathbb{C}^{2}, 0\right), \gamma(u)=\left(\gamma_{1}(u), \gamma_{2}(u)\right)$ be an irreducible complex plane curve germ. Define $F_{\gamma}:(\mathbb{C} \times \mathbb{C}, 0) \rightarrow\left(\mathbb{C}^{2}, 0\right)$ as in (3.1); that is,

$$
F_{\gamma}(u, v)=\left(\frac{\operatorname{det}\left(\gamma^{\prime}(u), \gamma(u)-\gamma(v)\right)}{(u-v)^{2}}, \frac{\operatorname{det}\left(\gamma^{\prime}(v), \gamma(u)-\gamma(v)\right)}{(u-v)^{2}}\right) .
$$

Definition 4.1. Let $\gamma$ be defined as above.

$$
\vartheta:=\operatorname{dim}_{\mathbb{C}} \frac{\mathcal{O}_{\mathbb{C}^{2}, 0}}{\left\langle F_{\gamma}(u, v)\right\rangle} \quad \text { and } \quad \tilde{\vartheta}:=\operatorname{dim}_{\mathbb{C}} \frac{\mathbb{C}[u, v]}{\left\langle F_{\gamma}(u, v)\right\rangle} .
$$


By Definition 1.1. $I$ and $\delta$ are the number of inflection points and of double points of a deformation of the curve, respectively. It follows from the complex version of Proposition 3.3 that

$$
\vartheta=2 T+2 \delta+I,
$$

where $T$ is the number of bitangencies of a deformation of the curve, not counting the sign. Analogously, for the corresponding global notions, we have

$$
\tilde{\vartheta}=2 \tilde{T}+2 \tilde{\delta}+\tilde{I},
$$

where $\tilde{T}$ is equal to $T$ plus the number of bitangencies of the curve before the deformation.

Lemma 4.2. Let $\alpha:(\mathbb{C}, 0) \rightarrow\left(\mathbb{C}^{2}, 0\right)$ be a germ of the type

$$
\alpha(u)=\left(a_{k} u^{k}, b_{m} u^{m}\right),
$$

where $0<k<m, a_{k} b_{m} \neq 0$ with $k$ and $m$ co-prime. Then $F_{\alpha}$ has the origin as an isolated zero in $\mathbb{C}$.

Proof. The vector field $F_{\alpha}$ can be written as

$$
\begin{aligned}
& F_{\alpha}(u, v)=\frac{a_{k} b_{m}}{(u-v)^{2}}\left((k-m) u^{k+m-1}\right.+m u^{m-1} v^{k}-k u^{k-1} v^{m}, \\
&\left.(m-k) v^{k+m-1}-m v^{m-1} u^{k}+k v^{k-1} u^{m}\right) .
\end{aligned}
$$

It is simple to see that $(0,0)$ is a zero of $F_{\alpha}$. We now study the zeros of $F_{\alpha}$ different from the origin. As $F_{\alpha}$ is homogeneous, if $(u, v) \neq(0,0)$ is a zero of $F_{\alpha}$, then all points on the straight line $(t u, t v)$ are zeros too. Suppose $(u, v) \neq(0,0)$ and define $X=v / u$. Thus

$$
F_{\alpha}(u, v)=(0,0) \Leftrightarrow F_{\alpha}(1, X)=(0,0) .
$$

Thus it is enough to find the zeros of the system

$$
\left\{\begin{array}{l}
\frac{-k X^{m}+m X^{k}+(k-m)}{(1-X)^{2}}=0 \\
\frac{(m-k) X^{m}-m X^{m-k}+k}{(1-X)^{2}}=0 .
\end{array}\right.
$$

Suppose that $z \in \mathbb{C}$ with $z \neq 0$ is a solution of (4.4). It follows by the first equation of (4.4) that

$$
z^{m}=\frac{m z^{k}+(k-m)}{k} .
$$

Substituting the value of $z$ into the second one, we have

$$
\begin{gathered}
(m-k)\left(\frac{m z^{k}+(k-m)}{k}\right)-m\left(\frac{m z^{k}+(k-m)}{k z^{k}}\right)+k=0 \\
\Leftrightarrow \frac{(m-k) m}{k}\left(z^{k}+\frac{1}{z^{k}}-2\right)=0 \\
\Leftrightarrow z^{k}+\frac{1}{z^{k}}-2=0 \\
\Leftrightarrow\left(z^{k}-1\right)^{2}=0 .
\end{gathered}
$$


Therefore $z^{k}=1$, so $z=e^{i \frac{2 \pi N}{k}}, N=0, \ldots, k-1$. Substituting these values in the first equation of (4.4), we have

$$
-k\left(e^{i \frac{2 \pi N}{k}}\right)^{m}+k=0 \Leftrightarrow e^{i \frac{2 \pi m N}{k}}=1 .
$$

By assumption $k$ and $m$ are co-prime, so the system (4.4) has no solutions other than $X=1$.

Proposition 4.3. Let $\gamma:(\mathbb{C}, 0) \rightarrow\left(\mathbb{C}^{2}, 0\right)$ be a germ of the type

$$
\gamma(u)=\left(\sum_{i=k}^{l} a_{i} u^{i}, \sum_{j=m}^{n} b_{j} u^{j}\right)
$$

with $a_{k} a_{l} b_{m} b_{n} \neq 0$ and $1 \leq k<m$. Then:

i) If $k$ and $m$ are co-prime, then $\vartheta=I^{2}$.

ii) If $l$ and $n$ are co-prime, then $\tilde{\vartheta}=\tilde{I}^{2}$.

Proof. Consider again $F_{\gamma}(u, v)=(f(u, v),-f(v, u))$, where $f(u, v)=\operatorname{det}\left(\gamma^{\prime}(u), \gamma(u)\right.$ $-\gamma(v)) /(u-v)^{2}$. From the comments of Subsection 2.2 it follows that to calculate a dimension of $\mathcal{O}_{\mathbb{C}^{2}, 0} /\left\langle F_{\gamma}\right\rangle$ it is sufficient to consider $\gamma$ as in (4.3). We have

$$
\begin{aligned}
& f(u, v)(u-v)^{2}=\left(\begin{array}{cc}
k a_{k} u^{k-1} & m b_{m} u^{m-1} \\
a_{k}\left(u^{k}-v^{k}\right) & b_{m}\left(u^{m}-v^{m}\right)
\end{array}\right) \\
&= k a_{k} b_{m} u^{k-1}\left(u^{m}-v^{m}\right)-m a_{k} b_{m} u^{m-1}\left(u^{k}-v^{k}\right) \\
&= a_{k} b_{m}(u-v)\left(k u^{k-1}\left(u^{m-1}+u^{m-2} v+\cdots+v^{m-1}\right)\right. \\
&\left.\quad-m u^{m-1}\left(u^{k-1}+u^{k-2} v+\cdots+v^{k-1}\right)\right) \\
&= a_{k} b_{m}(u-v)\left((k-m) u^{k+m-2}+(k-m) u^{k+m-3} v\right. \\
&\left.+\cdots+(k-m) u^{m-1} v^{k-1}+k u^{k-1}\left(u^{m-k-1} v^{k}+\cdots+v^{m-1}\right)\right) \\
&= a_{k} b_{m}(u-v)^{2}\left((k-m) u^{k+m-3}+v g(u, v)\right), \operatorname{ord}(g)=k+m-2 .
\end{aligned}
$$

Thus, $f$ is a homogeneous polynomial of degree $k+m-3$ given by $f(u, v)=$ $a_{k} b_{m}(k-m) u^{k+m-3}+v g(u, v)$ where $\operatorname{ord}(g)=k+m-2$. Since $k$ and $m$ are coprime, it follows from Lemma 4.2 that the origin is an isolated zero of $F_{\gamma}$. Therefore

$$
\begin{aligned}
\vartheta & =\operatorname{dim}_{\mathbb{C}} \frac{\mathcal{O}_{\mathbb{C}^{2}, 0}}{\left\langle F_{\gamma}(u, v)\right\rangle}=\operatorname{dim}_{\mathbb{C}} \frac{\mathcal{O}_{\mathbb{C}^{2}, 0}}{\langle f(u, v),-f(v, u)\rangle} \\
& =\operatorname{dim}_{\mathbb{C}} \frac{\mathcal{O}_{\mathbb{C}^{2}, 0}}{\left\langle a_{k} b_{m}(k-m) u^{k+m-3}+v g(u, v),-a_{k} b_{m}(k-m) v^{k+m-3}-u g(v, u)\right\rangle} \\
& =\operatorname{dim}_{\mathbb{C}} \frac{\mathcal{O}_{\mathbb{C}^{2}, 0}}{\left\langle u^{k+m-3}, v^{k+m-3}\right\rangle}=(k+m-3)^{2} .
\end{aligned}
$$

On the other hand, it is easy to see that

$I=\operatorname{dim}_{\mathbb{C}} \frac{\mathcal{O}_{\mathbb{C}^{2}, 0}}{\left\langle\operatorname{det}\left(\gamma^{\prime}(u), \gamma^{\prime \prime}(u)\right)\right\rangle}=\operatorname{dim}_{\mathbb{C}} \frac{\mathcal{O}_{\mathbb{C}^{2}, 0}}{\left\langle k m(m-k) a_{k} b_{m} u^{k+m-3}+\cdots\right\rangle}=(k+m-3)$. 
ii) In the notation of Section 2 consider

$F_{\gamma}(u, v)=\left(P_{k+m-3}(u, v)+\cdots+P_{l+n-3}(u, v),-P_{k+m-3}(v, u)-\cdots-P_{l+n-3}(v, u)\right)$, where $P_{j}$ is a homogeneous polynomial of degree $j$.

Writing

$$
\begin{aligned}
F_{1}(w, u, v)= & w^{l+n-k-m} P_{k+m-3}(u, v)+w^{l+n-k-m-1} P_{k+m-2}(u, v) \\
& +\cdots+P_{l+n-3}(u, v), \\
F_{2}(w, u, v)= & -w^{l+n-k-m} P_{k+m-3}(v, u)-w^{l+n-k-m-1} P_{k+m-2}(v, u) \\
& -\cdots-P_{l+n-3}(v, u),
\end{aligned}
$$

we have that

$$
\begin{aligned}
& F_{\gamma}(u, v)=\left(F_{1}(1, u, v), F_{2}(1, u, v)\right)=\left(f_{1}(u, v), f_{2}(u, v)\right), \\
& \bar{F}_{1}(u, v)=F_{1}(0, u, v)=P_{l+n-3}(u, v), \\
& \bar{F}_{2}(u, v)=F_{2}(0, u, v)=-P_{l+n-3}(v, u) .
\end{aligned}
$$

By hypothesis, $l$ and $n$ are co-prime. It follows by Lemma 4.2 that $\bar{F}_{1}=\bar{F}_{2}=0$ has the origin as an isolated zero. Therefore $f_{1}=f_{2}=0$ has no solutions at infinity. It follows by Bézout's Theorem that

$$
\tilde{\vartheta}=\operatorname{dim}_{\mathbb{C}} \frac{\mathbb{C}[u, v]}{\left\langle F_{\gamma}(u, v)\right\rangle}=\operatorname{dim}_{\mathbb{C}} \frac{\mathbb{C}[u, v]}{\left\langle f_{1}(u, v), f_{2}(u, v)\right\rangle}=(l+n-3)^{2} .
$$

On the other hand, it is easy to see that

$$
\tilde{I}=\operatorname{dim}_{\mathbb{C}} \frac{\mathbb{C}[u]}{\left\langle\operatorname{det}\left(\gamma^{\prime}(u), \gamma^{\prime \prime}(u)\right)\right\rangle}=(l+n-3) .
$$

The proposition is proved.

We know that $\delta$ can be calculated in many different ways. For example, Milnor showed in [13] that if the image of the map germ $g$ has defining equation $G \in \mathcal{O}_{\mathbb{C}^{2}, 0}$, then the local Milnor number

$$
\mu=\operatorname{dim}_{\mathbb{C}} \frac{\mathcal{O}_{\mathbb{C}^{2}, 0}}{\left\langle\frac{\partial G}{\partial x}, \frac{\partial G}{\partial y}\right\rangle}
$$

of $G$ is related to $\delta$ by the formula $\mu=2 \delta-r+1$, where $r$ and $\delta$ are the number of branches of the curve and the number of nodes appearing in a stable perturbation, respectively. In the case of germs $(r=1)$, we have $\mu=2 \delta$. The global Milnor number, which we denote by $\tilde{\mu}$, is the sum of all the local Milnor numbers at all the critical points of $G$. Thus, we have the following:

Theorem 4.4. Let $\gamma:(\mathbb{C}, 0) \rightarrow\left(\mathbb{C}^{2}, 0\right)$ be a germ of the type

$$
\gamma(u)=\left(\sum_{i=k}^{l} a_{i} u^{i}, \sum_{j=m}^{n} b_{j} u^{j}\right)
$$

with $a_{k} a_{l} b_{m} b_{n} \neq 0$ and $1 \leq k<m$. Then:

i) If $k$ and $m$ are co-prime, then

$$
\mu=I(I-1)-2 T \text {. }
$$

ii) If $l$ and $n$ are co-prime, then

$$
\tilde{\mu}=\tilde{I}(\tilde{I}-1)-2 \tilde{T} .
$$


Proof. From equations (4.1) and (4.2) we have

$$
\vartheta=2 T+2 \delta+I \quad \text { and } \quad \tilde{\vartheta}=2 \tilde{T}+2 \tilde{\delta}+\tilde{I} .
$$

From these equations and Proposition 4.3 we obtain the following formulae:

$$
I(I-1)=2 T+2 \delta \quad \text { and } \quad \tilde{I}(\tilde{I}-1)=2 \tilde{T}+2 \tilde{\delta},
$$

relating inflections, bitangencies and a double point of plane curve germs. Now the proof follows directly from the above comments.

In particular, constancy of the invariants $I$ and $T$ in this setting implies constancy of the Milnor number.

Let $\mathcal{O}_{n, p}$ be the vector space of map germs $f:\left(\mathbb{C}^{n}, 0\right) \rightarrow\left(\mathbb{C}^{p}\right)$. When $p=1$, $\mathcal{O}_{n, 1}=\mathcal{O}_{n}$ is the local ring of germs of functions in $n$-variables. The set $\mathcal{O}_{n, p}$ is a free $\mathcal{O}_{n}$-module of rank $p$. Let $\theta_{\mathbb{C}^{n}, 0}$ and $\theta_{\mathbb{C}^{p}, 0}$ be the $\mathcal{O}_{n}$-module of germs at 0 of vector fields on $\mathbb{C}^{n}$ and $\mathcal{O}_{p}$-module of germs at 0 of vector fields on $\mathbb{C}^{p}$ respectively. Let $\theta(f)$ be the $\mathcal{O}_{n}$-module of germs $\xi:\left(\mathbb{C}^{n}, 0\right) \rightarrow T \mathbb{C}^{p}$ such that $\pi_{p} \circ \xi=f$, where $\pi_{p}: T \mathbb{C}^{p} \rightarrow \mathbb{C}^{p}$ denotes the tangent bundle over $\mathbb{C}^{p}$.

Define $t f: \theta_{\mathbb{C}^{n}, 0} \rightarrow \theta(f)$ by $t f(\chi)=d f \circ \chi$ and $w f: \theta_{\mathbb{C}^{p}, 0} \rightarrow \theta(f)$ by $w f(\eta)=\eta \circ f$. The $\mathcal{A}_{e}$-tangent space of $f$ is defined as $T \mathcal{A}_{e} f=\operatorname{tf}\left(\theta_{\mathbb{C}^{n}, 0}\right)+w f\left(\theta_{\mathbb{C}^{p}, 0}\right)$. Finally we define the $\mathcal{A}_{e}$-codimension of a germ $f$, denoted by $\mathcal{A}_{e}$-cod $(f)$, as the $\mathbb{C}$-vector space dimension of

$$
N A_{e}(f)=\frac{\theta(f)}{T \mathcal{A}_{e} f}
$$

Two $\mathcal{A}$-equivalent curves have equal Milnor number ([1]; check this reference for more details about $\mathcal{A}$-equivalence too).

Theorem 4.5 ([14]). Let $\gamma:(\mathbb{C}, 0) \rightarrow\left(\mathbb{C}^{2}, 0\right)$ be $\mathcal{A}$-finitely determined plane curve germ. Then $\mu \geq 2 \mathcal{A}_{e}-\operatorname{cod} \gamma$ with equality if and only if $\gamma$ is quasihomogeneous.

Corollary 4.6. Let $\gamma$ be as in (4.5) with $k$ and $m$ co-prime. Then

$$
I(I-1)-2 T \geq 2 \mathcal{A}_{e}-\operatorname{cod} \gamma,
$$

with equality if and only if $\gamma$ is quasihomogeneous.

In Table 1 we calculate the invariants $I, \delta, T, \vartheta$ and $\mu$ for some simple germs of plane curves classified in [2, and in Table 2 we calculate the invariants $\tilde{I}, \tilde{\delta}, \tilde{T}, \tilde{\vartheta}$ and $\tilde{\mu}$ for those same germs.

TABLE 1. Local invariants for simple germs of plane curves.

\begin{tabular}{|c|c|c|c|c|c|c|}
\hline Name & Normal Form & $I$ & $\delta$ & $T$ & $\vartheta$ & $\mu$ \\
\hline & $\left(t, t^{k}\right)$ & $k-2$ & 0 & $\frac{(k-2)(k-3)}{2}$ & $(k-2)^{2}$ & 0 \\
\hline$A_{2 k}$ & $\left(t^{2}, t^{2 k+1}\right)$ & $2 k$ & $k$ & $2 k(k-1)$ & $4 k^{2}$ & $2 k$ \\
\hline$E_{6 k}$ & $\left(t^{3}, t^{3 k+1}\right), k \geq 1$ & $3 k+1$ & $3 k$ & $\frac{3 k(3 k-1)}{2}$ & $(3 k+1)^{2}$ & $6 k$ \\
\hline$E_{6 k+2}$ & $\left(t^{3}, t^{3 k+2}\right), k \geq 1$ & $3 k+2$ & $3 k+1$ & $\frac{3 k(3 k+1)}{2}$ & $(3 k+2)^{2}$ & $6 k+2$ \\
\hline$W_{12}$ & $\left(t^{4}, t^{5}\right) ;\left(t^{4}, t^{5}+t^{7}\right)$ & 6 & 6 & 9 & 36 & 12 \\
\hline$W_{18}$ & $\left(t^{4}, t^{7}\right) ;\left(t^{4}, t^{7}+t^{9}\right) ;\left(t^{4}, t^{7}+t^{13}\right)$ & 8 & 9 & 19 & 64 & 18 \\
\hline
\end{tabular}


TABLE 2. Global invariants for simple germs of plane curves.

\begin{tabular}{|c|c|c|c|c|c|c|}
\hline Name & Normal Form & $\tilde{I}$ & $\tilde{\delta}$ & $\tilde{T}$ & $\tilde{\vartheta}$ & $\tilde{\mu}$ \\
\hline & $\left(t, t^{k}\right)$ & $k-2$ & 0 & $\frac{(k-2)(k-3)}{2}$ & $(k-2)^{2}$ & 0 \\
\hline$A_{2 k}$ & $\left(t^{2}, t^{2 k+1}\right)$ & $2 k$ & $k$ & $2 k(k-1)$ & $4 k^{2}$ & $2 k$ \\
\hline$E_{6 k}$ & $\left(t^{3}, t^{3 k+1}\right), k \geq 1$ & $3 k+1$ & $3 k$ & $\frac{3 k(3 k-1)}{2}$ & $(3 k+1)^{2}$ & $6 k$ \\
\hline$E_{6 k+2}$ & $\left(t^{3}, t^{3 k+2}\right), k \geq 1$ & $3 k+2$ & $3 k+1$ & $\frac{3 k(3 k+1)}{2}$ & $(3 k+2)^{2}$ & $6 k+2$ \\
\hline$W_{12}$ & $\left(t^{4}, t^{5}\right)$ & 6 & 6 & 9 & 36 & 12 \\
\hline$W_{12}$ & $\left(t^{4}, t^{5}+t^{7}\right)$ & 8 & 9 & 19 & 64 & 18 \\
\hline$W_{18}$ & $\left(t^{4}, t^{7}\right)$ & 8 & 9 & 19 & 64 & 18 \\
\hline$W_{18}$ & $\left(t^{4}, t^{7}+t^{9}\right)$ & 10 & 12 & 33 & 100 & 24 \\
\hline$W_{18}$ & $\left(t^{4}, t^{7}+t^{13}\right)$ & 14 & 18 & 73 & 196 & 36 \\
\hline$W_{1,2 k-1}^{\sharp}$ & $\left(t^{4}, t^{6}+t^{2 k+5}\right), k \geq 1$ & $2 k+6$ & $3(k+2)$ & $2 k^{2}-14 k+21$ & $(2 k+6)^{2}$ & $6(k+2)$ \\
\hline
\end{tabular}

Example 4.7. Consider two $\mathcal{A}$-equivalent germs $(\mathbb{C}, 0) \rightarrow\left(\mathbb{C}^{2}, 0\right)$ given by

$$
t \mapsto\left(t^{2}, t^{5}\right) \text { and } t \mapsto\left(t^{2}, t^{4}+t^{5}\right) .
$$

We show in Table 3 the values for the different invariants. It can be seen that both $I$ and $T$ are different for these two germs. However, in [5] and later in [15] the notion of $\mathcal{A}_{h}$-equivalence is introduced. It is a finer version of $\mathcal{A}$-equivalence which preserves the flat geometry (i.e. inflections). Therefore, $\mathcal{A}_{h}$-equivalent germs have equal values for $I$ and $T$.

TABLE 3. Ramphoid cusp.

\begin{tabular}{|c|c|c|c|c|c|}
\hline Normal Form & $I \backslash \tilde{I}$ & $\delta \backslash \tilde{\delta}$ & $T \backslash \tilde{T}$ & $\vartheta \backslash \tilde{\vartheta}$ & $\mu \backslash \tilde{\mu}$ \\
\hline$\left(t^{2}, t^{5}\right)$ & $4 \backslash 4$ & $2 \backslash 2$ & $4 \backslash 4$ & $16 \backslash 16$ & $4 \backslash 4$ \\
\hline$\left(t^{2}, t^{4}+t^{5}\right)$ & $3 \backslash 4$ & $2 \backslash 2$ & $2 \backslash 4$ & $11 \backslash 16$ & $4 \backslash 4$ \\
\hline
\end{tabular}

Finally we make some considerations about the case where there is more than one branch involved. Consider the plane curve germ

$$
\gamma(u)=\left\{\gamma_{1}(u), \gamma_{2}(u), \ldots, \gamma_{r}(u)\right\}
$$

with $r$ branches. Denote by

i) $\delta_{i j}$ the number of points $(u, v)$ such that $\gamma_{i}(u)=\gamma_{j}(v)$ with $i \neq j$.

ii) $T_{i j}$ the number of points $(u, v)$ such that $\gamma_{i}^{\prime}(u) \|\left(\gamma_{i}(u)-\gamma_{j}(v)\right)$ and $\gamma_{i}^{\prime}(u) \|$ iii) $\gamma_{j}^{\prime}(v)$ with $i \neq j$.

$$
\vartheta_{i j}:=\operatorname{dim}_{\mathbb{C}} \frac{\mathcal{O}_{\mathbb{C}^{2}, 0}}{\left\langle\operatorname{det}\left(\gamma_{i}^{\prime}(u), \gamma_{i}(u)-\gamma_{j}(v)\right), \operatorname{det}\left(\gamma_{j}^{\prime}(v), \gamma_{i}(u)-\gamma_{j}(v)\right)\right\rangle} .
$$

iv)

$$
\delta=\sum_{1 \leq i<j \leq r} \delta_{i j}+\sum_{i=1}^{r} \delta_{i}
$$

Therefore $\vartheta_{i j}=\delta_{i j}+T_{i j}$. 
Remark 4.8. Let $\gamma$ be as in (4.6). Thus from the proof of Theorem 4.4 and the above comments we have

$$
\begin{aligned}
\mu+r-1=2 \delta & =2\left(\sum_{1 \leq i<j \leq r} \delta_{i j}+\sum_{i=1}^{r} \delta_{i}\right) \\
& =2 \sum_{1 \leq i<j \leq r} \delta_{i j}+\sum_{i=1}^{r}\left(I_{i}\left(I_{i}-1\right)-2 T_{i}\right) .
\end{aligned}
$$

\section{ACKNOWLEDGEMENTS}

This paper was written during the postdoctoral programs of the first and second authors at the Instituto de Ciências Matemáticas e de Computação, USP, São Carlos, Brazil. The authors are grateful to J. J. Ñuno Ballesteros for useful discussions.

\section{REFERENCES}

[1] V. I. Arnol'd, S. M. Gusel̆n-Zade, and A. N. Varchenko, Singularities of differentiable maps. Vol. I, The classification of critical points, caustics and wave fronts. Translated from the Russian by Ian Porteous and Mark Reynolds, Monographs in Mathematics, vol. 82, Birkhäuser Boston Inc., Boston, MA, 1985. MR777682 (86f:58018)

[2] J. W. Bruce and T. J. Gaffney, Simple singularities of mappings $\mathbf{C}, 0 \rightarrow \mathbf{C}^{2}, 0$, J. London Math. Soc. (2) 26 (1982), no. 3, 465-474, DOI 10.1112/jlms/s2-26.3.465. MR684560 (84d:32012)

[3] David Cox, John Little, and Donal O'Shea, Using algebraic geometry, Graduate Texts in Mathematics, vol. 185, Springer-Verlag, New York, 1998. MR.1639811 (99h:13033)

[4] Fabio Scalco Dias and Luis Fernando Mello, Geometry of plane curves, Bull. Sci. Math. 135 (2011), no. 4, 333-344, DOI 10.1016/j.bulsci.2011.03.007. MR2799811(2012f:53003)

[5] F. S. Dias and J. J. Nuño-Ballesteros, Plane curve diagrams and geometrical applications, Q. J. Math. 59 (2008), no. 3, 287-310, DOI 10.1093/qmath/ham039. MR2444062 (2009m:58087)

[6] Freddy Dumortier, Jaume Llibre, and Joan C. Artés, Qualitative theory of planar differential systems, Universitext, Springer-Verlag, Berlin, 2006. MR2256001 (2007f:34001)

[7] Fr. Fabricius-Bjerre, On the double tangents of plane closed curves, Math. Scand 11 (1962), 113-116. MR0161231 (28 \#4439)

[8] Fr. Fabricius-Bjerre, A relation between the numbers of singular points and singular lines of a plane closed curve, Math. Scand. 40 (1977), no. 1, 20-24. MR0444673 (56 \#3023)

[9] Emmanuel Ferrand, On the Bennequin invariant and the geometry of wave fronts, Geom. Dedicata 65 (1997), no. 2, 219-245, DOI 10.1023/A:1004936711196. MR.1451976 (99c:57061)

[10] Benjamin Halpern, Global theorems for closed plane curves, Bull. Amer. Math. Soc. 76 (1970), 96-100. MR0262936 (41 \#7541)

[11] Morris W. Hirsch, Differential topology, Graduate Texts in Mathematics, No. 33, SpringerVerlag, New York, 1976. MR0448362 (56 \#6669)

[12] John W. Milnor, Topology from the differentiable viewpoint, based on notes by David W. Weaver, The University Press of Virginia, Charlottesville, Va., 1965. MR 0226651 (37 \#2239)

[13] John Milnor, Singular points of complex hypersurfaces, Annals of Mathematics Studies, No. 61, Princeton University Press, Princeton, N.J.; University of Tokyo Press, Tokyo, 1968. MR0239612 (39 \#969)

[14] David Mond, Looking at bent wires- $\mathcal{A}_{e}$-codimension and the vanishing topology of parametrized curve singularities, Math. Proc. Cambridge Philos. Soc. 117 (1995), no. 2, 213-222, DOI 10.1017/S0305004100073060. MR.1307076 (95m:58017)

[15] R. Oset Sinha and F. Tari. Projections of space curves and duality, Q. J. Math. 64 (2013), no. 1, 281-302. MR3032100 
[16] C. T. C. Wall, Singular points of plane curves, London Mathematical Society Student Texts, vol. 63, Cambridge University Press, Cambridge, 2004. MR2107253 (2005i:14031)

[17] Joel L. Weiner, A spherical Fabricius-Bjerre formula with applications to closed space curves, Math. Scand. 61 (1987), no. 2, 286-291. MR.947479 (89h:53004)

Instituto de Ciências Exatas, Universidade Federal de Itajubá, Avenida BPS 1303, Pinheirinho, CEP 37.500-903, ItajubÁ, MG, Brazil

E-mail address: scalco@unifei.edu.br

Departamento de Matemática, Instituto de Ciências Matemáticas e de ComputaÇão, Universidade de São Paulo, CEP 13.560-970, São Carlos-SP, Brazil

E-mail address: raul.oset@uv.es

Departamento de Matemática, Instituto de Ciências Matemáticas e de ComputaÇão, Universidade de SÃo Paulo, CEP 13.560-970, SÃo Carlos-SP, Brazil

E-mail address: maasruas@icmc.usp.br 